\title{
THEORETICAL INVESTIGATION OF THE $C-V$ RELATIONSHIP FOR AN AMORPHOUS SILICON $p-n$ JUNCTION
}

\author{
Hsiung-Kuang Tsai and SI-Chen LeE \\ Department of Electrical Engineering, National Taiwan University, Taipei, Taiwan, Republic of China
}

(Received 24 October 1988; in revised form 17 March 1989)

\begin{abstract}
The use of capacitance-voltage measurement to probe the continuous gap state distribution of hydrogenated amorphous silicon is reconsidered. Since the capacitance is measured by a small a.c. voltage, whereas the data is plotted against d.c. voltage, care must be taken in extracting the gap state density from the slope of the capacitance-voltage curve. It is found that under large reverse bias, the $1 / C^{2}-V$ curve of an a-Si: $\mathrm{H}^{+}-n$ junction shows a linear relation, similar to a crystal junction, and that the density obtained is the total gap state charge density.
\end{abstract}

\section{INTRODUCTION}

Since the first introduction of junction capacitancevoltage $(C-V)$ measurements in characterizing the semiconductor gap states[1], the capacitance method has been developed to many different variations such as $C-T, C \dashv \omega, C-T \dashv \omega$ and transient measurements[2], where $T, \omega$ are temperature and frequency, respectively. These methods were applied to crystalline as well as to amorphous junctions to obtain information about the density of the shallow impurity states or gap state distribution. However, due to the presence of a continuous gap state distribution, the theoretical analysis for an amorphous junction is far more difficult than the crystal case.

Among these studies, analysis of the $C-V$ measurement was almost always based on the results of Roberts and Crowell[3], which required all the deep states to respond to the measuring a.c. signal. This assumption is questionable for hydrogenated amorphous silicon $(\mathrm{a}-\mathrm{Si}: \mathrm{H})$ junction, since no matter how low the frequency is, say $0.1 \mathrm{~Hz}$, there are still some states which cannot respond[4]. Lang et al.[5] have noted this difference, namely that the capacitance is the measure of a specific width within the depletion region depending on the frequency of the a.c. measuring voltage while the total depletion width is determined by the d.c. bias voltage. This difference suggests that the gap state density obtained from the $C-V$ curve is determined by those states that can follow the bias voltage. However, to our knowledge, no explicit formulation has been derived. In this paper, we will present a detailed analysis of the $C-V$ measurement of an a-Si: $\mathrm{H}^{+}-n$ junction and show that the deep levels could be probed by varying the bias voltage, not the a.c. measuring voltage.

In order to derive the $C-V$ relation, the charge distribution in the depletion region must be first determined. This distribution, $\rho(x)$, is determined by the density of the gap states, $N(E)$, and the electron occupation probability, $f(E, x)$, at different biases and can be expressed as:

$$
\rho(x)=\int_{E_{\mathrm{v}}}^{E_{\mathrm{c}}} N(E)\left[f(E, x)-f_{0}(E, x)\right] \mathrm{d} E,
$$

where $f_{0}(E, x)$ is the occupation probability at equilibrium, $x$ is the position. Although the density of gap states is strongly affected by the preparation conditions and is not known in all cases, the occupation probability can still be determined. In the following, we shall first use the Shockley-Read-Hall (SRH) recombination model[6,7] to determine the electron occupation probability $f(E, x)$ of the same deep states in three different conditions that will be present in a reverse biased junction. Then the carrier transport equations are investigated self-consistently to determine how these three conditions are met in the depletion region. With this occupation probability and the assumption of a uniform media, the charge distribution can be obtained.

\section{THEORY}

According to the SRH model, the electron occupation probability of an gap state with energy level, $E$, can be written as[8]:

$$
f(E, x)=\frac{n+b \bar{p}}{n+\bar{n}+b(p+\bar{p})},
$$

where $p$ and $n$ are the free hole and free electron concentration at position $x$, respectively, which also represent the hole and electron capture rate of the state at $E \cdot \bar{p}$ and $\bar{n}$ for a donor-type gap state are given by:

$$
\begin{aligned}
& \bar{n}=\frac{N_{\mathrm{c}}}{g} \exp \left(\frac{E-E_{\mathrm{c}}}{k T}\right) \\
& \bar{p}=g N_{\mathrm{v}} \exp \left(\frac{E_{\mathrm{v}}-E}{k T}\right)
\end{aligned}
$$


which are related to the emission rate of the state $E$, where $g$ is the degeneracy factor, $E_{\mathrm{c}}$ and $E_{\mathrm{v}}$ the respective conduction and valence band mobility edges, $N_{\mathrm{c}}$ and $N_{\mathrm{v}}$ the corresponding effective densities of states, $b=\sigma_{p} v_{p} / \sigma_{n} v_{n}$, where $\sigma_{p}\left(\sigma_{n}\right)$ and $v_{p}\left(v_{n}\right)$ are the hole (electron) capture cross-section and the thermal velocity, respectively. For an accepter-type gap state, the $g$ factors in the formula for $\bar{n}$ and $\bar{p}$ are replaced by $1 / \mathrm{g}$. Since the emission rate of a gap state is dependent on its energy, a special energy, $E_{\mathrm{i}}^{\prime}$, at which the state has equal electron and hole emission rate, i.e. $\bar{n}=b \bar{p}$, can be defined:

$$
E_{\mathrm{i}}^{\prime}=\frac{E_{\mathrm{c}}+E_{\mathrm{v}}}{2}+\frac{k T}{2} \ln \left(\frac{g^{2} b N_{\mathrm{v}}}{N_{\mathrm{c}}}\right),
$$

which is close to the intrinsic Fermi level, $E_{\mathrm{i}}$, differing only by a small quantity, $k T / 2 \ln \left(g^{2} b\right)$. Now we shall consider $f(E, x)$ at three different locations inside the depletion region in a reverse-biased $p n$ junction. Typically, under reverse bias, the hole quasi-Fermi level $E_{f p}$ is situated always above the electron quasiFermi level $E_{f n}$. Figures 1 (a), (b) and (c) show the relative positions of $E_{f n}, E_{f p}$ and $E_{\mathrm{i}}^{\prime}$ in the gap. When $E_{f n}$ and $E_{f p}$ are all above (below) $E_{\mathrm{i}}^{\prime}$, as shown in Fig. 1(a) (Fig. 1b), $f(E, x)$ can be estimated from eqn (2) which turns out to approximately equal to 1 for $E$ less than $E_{f_{n}}\left(E_{f_{p}}\right)$. Thus, in these two cases, the electron occupation probability of the gap state is
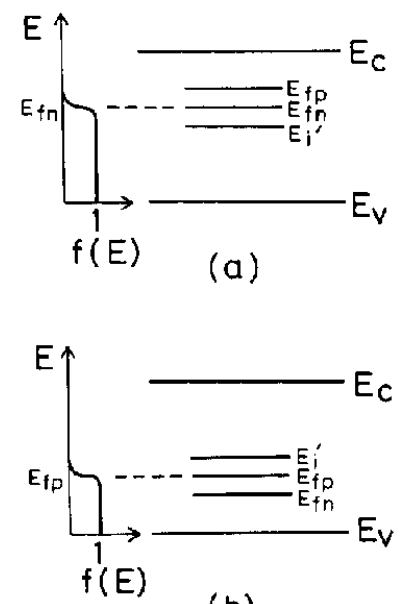

(b)

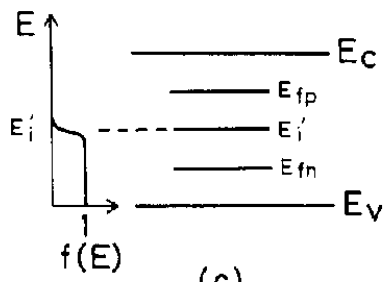

(c)

Fig. 1. Three different conditions that will present in a reverse biased junction. The electron and hole capture rates are significant in (a) and (b), respectively, and the emission rate is dominant in (c). The electron occupation probability, $f(E)$, in each case are also shown. determined by the majority carrier quasi-Fermi level which depends on the external bias. On the other hand, when $E_{\mathrm{i}}^{\prime}$ is located in between $E_{\mathrm{f} n}$ and $E_{\mathrm{f}_{p}}$ (Fig. $1 \mathrm{c}), f(E, x)$ is determined by $E_{\mathrm{i}}^{\prime}$ which is soley dependent on the material properties and independent of the external bias. This suggests that, when the free carrier density is sufficiently small, the occupation probability of the gap state is determined by the emission rate of the states and independent of the applied bias[9].

Next, we shall use the continuity equation to derive the variation of $E_{\mathrm{f} n}$ and $E_{\mathrm{f} p}$, in a reverse biased $p^{+}-n$ junction. Considering both diffusion and drift currents, the electron current density can be written as[10]:

$$
J_{n}=n \mu_{n} \nabla E_{\mathrm{f} n},
$$

where $\mu_{n}$ is the electron mobility and $n$ is the electron concentration $n$ given by:

$$
n=N_{\mathrm{c}} \exp \left[-\left(E_{\mathrm{c}}-E_{\mathrm{in}}\right) / k T\right] .
$$

This equation can be integrated from the edge of the depletion layer $y=W$ to $x$ (Fig. 2a), i.e.

$$
\frac{\Delta E_{\mathrm{f} n}}{k T}=\ln \left(1+\int_{W}^{x} \frac{J_{n}}{n(W) \mu_{n} k T} \exp \left(\frac{\Delta E_{\mathrm{c}}(y)}{k T}\right) \mathrm{d} y\right),
$$

where $\Delta E_{\mathrm{fn}}=E_{\mathrm{f} n}(x)-E_{\mathrm{f} n}(W)$ and $\Delta E_{\mathrm{c}}(y)=E_{\mathrm{c}}(y)-$ $E_{\mathrm{c}}(W)$. In Fig. 2(a), a linear variation of the mobility edges $E_{\mathrm{c}}$ and $E_{\mathrm{v}}$ has been used. This is only for illustration purposes and irrelevant to the following discussions. The electron current density $J_{n}(x)$ in eqn (4) is not constant due to the generation process in the reverse-biased junction and can be obtained from the continuity equation:

$$
J_{n}(x)=J_{n}(W)-\int_{W}^{x} e G(y) \mathrm{d} y,
$$

where $G(y)(>0)$ is the net electron-hole generation rate in the depletion region.

According to eqn (4), $\Delta E_{\text {fn }}$ can be approximated by:

$$
\frac{\Delta E_{\text {fn }}}{k T}= \begin{cases}0 & \text { if } \quad \begin{array}{l}
A<1 \\
\ln (A)
\end{array} \quad A>1\end{cases}
$$

where

$$
A \equiv \int_{W}^{x} \frac{J_{n}}{n(W) \mu_{n} k T} \exp \left(\frac{\Delta E_{\mathrm{c}}(y)}{k T}\right) \mathrm{d} y .
$$

Although, it is difficult to carry out the integration analytically, however, we can simplify it by first considering a crystal junction under the same conditions. In that case, eqns (4), (6) and (7) can still be applied because up to now we do not restrict the derivation to crystal or amorphous junction. Then, $\Delta E_{\mathrm{c}}(x)$ can be obtained based on the depletion approximation:

$$
\Delta E_{\mathrm{c}}(x) / k T=[(x-W) / l]^{2} / 2,
$$

where $l\left[\equiv \sqrt{(\epsilon k T) /\left(q^{2} N_{\mathrm{D}}\right)}\right]$ is the Debye length. Since the most important contribution to the eqn ( 7 ) comes 


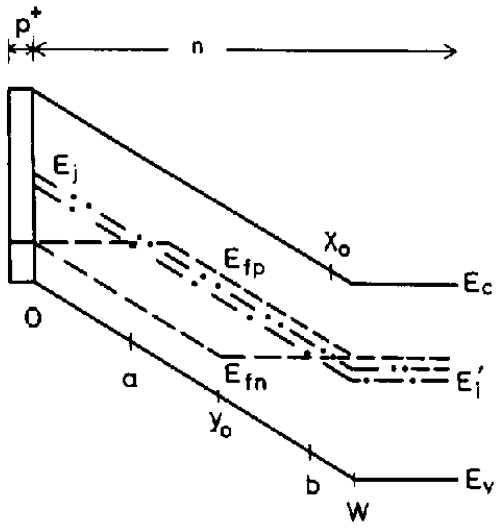

(a)

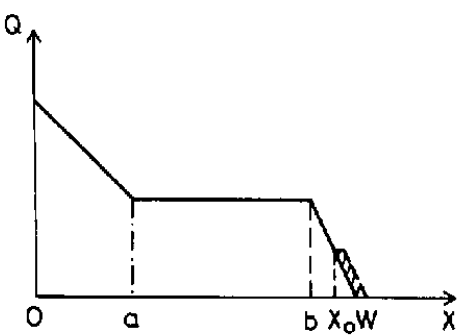

(b)

Fig. 2. A schematic diagram of (a) the energy diagram and (b) the charge distribution of a $p^{+}-n$ junction. The coordinates used in the text are also marked. The charge distribution was constant within $a \leqslant x \leqslant b$ and the hatched area indicated its change due to the a.c. signal.

from the position around $x$ and $J_{n}(x)$ is a slowly varying function as compared to $\exp \left(\Delta E_{\mathrm{c}} / k T\right)$, the integration can be approximated by multiplying the integrand at $x$ by a distance $l^{2} /(W-x)$ at which the integrand reduces to $36.8 \%\left(e^{-1}\right)$ of the maximum value, i.e.

$$
A \approx \frac{J_{n} l^{2}}{n(W)(W-x) \mu_{n} k T} \exp \left(\frac{\Delta E_{\mathrm{c}}(x)}{k T}\right) .
$$

Let $A$ equal to 1 at $y=y_{0}$, i.e.

$$
\Delta E_{c}\left(y_{0}\right) \equiv k T \ln \left(\frac{n(W)\left(W-y_{0}\right) \mu_{n} k T}{J_{n} l^{2}}\right),
$$

and substitute this back into eqns (6) and (7), $\Delta E_{\text {fn }}$ can be approximated by: junction is involved. Next, we should assume that the approximation can be applied to the amorphous junction and make a self-consistent check later.

Based on the above approximation, the magnitude of $\Delta E_{\mathrm{c}}\left(y_{0}\right)$ can be calculated. Assume the following parameters: $J_{n}=10 \mathrm{nA} / \mathrm{cm}^{2}, \quad \sigma_{n}=10^{-7}(\Omega-\mathrm{cm})^{-1}$, and $l=1000 \AA$,

$$
\Delta E_{\mathrm{c}}\left(y_{0}\right)=14 k T
$$

The position of $E_{\mathrm{i}}^{\prime}$ which is defined in eqn (3) and treated as the intrinsic Fermi level can be roughly estimated from the activation energy $(\sim 1.0 \mathrm{eV})$ of those lightly boron-doped a-Si:H that show the minimum dark conductivity. The separation between $E_{\mathrm{i}}^{\prime}$ and $E_{\mathrm{F}}$ of $n$-type a-Si: $\mathrm{H}$ can be readily obtained from its activation energy. The typical value for a $n$ layer doped with $1 \mathrm{ppm} P H_{3}\left(\sigma_{\mathrm{D}} \approx 10^{-7}(\Omega-\mathrm{cm})^{-1}\right)$ is approximately $10 k T$. A similar discussion can be applied to $E_{f_{p}}$. The resultant relative position between $E_{f n}, E_{f p}$ and $E_{\mathrm{i}}^{\prime}$ is shown in Fig. 2(a), and the crossover points between $E_{\mathrm{i}}^{\prime}$ and $E_{\mathrm{f} p}, E_{\mathrm{i}}^{\prime}$ and $E_{\mathrm{f} n}$ are defined to be $a$ and $b$, respectively. Following previous discussions, the electron occupation probability, $f(E, x)$, is determined by $E_{\mathrm{f} p}, E_{\mathrm{i}}^{\prime}$ and $E_{\mathrm{f} n}$ within $x \leqslant a, a \leqslant x \leqslant b$, and $b \leqslant x \leqslant W$, respectively.

The most important thing should be noted here is that in the region between $a$ and $b, f(E, x)$ is determined by $E_{\mathrm{i}}^{\prime}$ and independent of the position. If the gap state density is uniform, the space charge density in this region is also constant. Thus it is possible to define a density called "net gap state density" $N$ in this region, i.e. in the zero temperature approximation:

$$
N=\int_{E_{\mathrm{i}}^{\prime}}^{E_{\mathrm{F}}} N(E) \mathrm{d} E
$$

where $E_{\mathrm{F}}$ is the equilibrium Fermi-level position.

In the other two regions, however, no such simple results can be found, as $f(E, x)$ is determined by $E_{\text {f } n}$ and $E_{\mathrm{f} p}$, respectively. It is then natural to separate the depletion region into three parts in the following discussions. Figure 2(b) shows schematically the charge distribution (-) in each region under a bias $V$. Several properties of these regions should be noted. First, the electrostatic potential difference between $x=0$ and $x=a$ (region 1) is equal to $\left[E_{\mathrm{i}}^{\prime}(0)-E_{\uparrow p}(0)\right] / e\left(\equiv V_{p}\right)$ and holds constant at all

$$
\Delta E_{\text {fn }} \approx \begin{cases}0 & \text { if } x>y_{0} \\ \Delta E_{\mathrm{c}}(x)-\Delta E_{\mathrm{c}}\left(y_{0}\right)+k T \ln \left(\frac{J_{n}(x)\left(W-y_{0}\right)}{J_{n}\left(y_{0}\right)(W-x)}\right) & \text { if } x<y_{0}\end{cases}
$$

i.e. $E_{\mathrm{f} n}$ holds constant for $x$ larger than $y_{0}$ and almost parallel to $E_{\mathrm{c}}$ for $x$ smaller than $y_{0}$, as shown schematically in Fig. 2(a). This result, however, is solely dependent on the relative change of $\Delta E_{\mathrm{c}}$ and $J_{n}$ and is irrelevant to whether a crystal or amorphous biases. Similarly, the difference between $x=b$ and $x=W$ (region 3), $\left[E_{\mathrm{fn}}(W)-E_{\mathrm{i}}^{\prime}(W)\right] / e\left(\equiv V_{n}\right)$ also holds constant. Since the built-in potential, $V_{\mathrm{bi}}$, is equal to $V_{n}+V_{p}$, we conclude that all the external bias, $V$ is applied over the constant charge region 
from $x=a$ to $x=b$ (region 2), i.e.

$$
V=\frac{q N}{2 \epsilon}(b-a)^{2}+\left.\frac{\mathrm{d} \phi}{\mathrm{d} x}\right|_{b}(b-a),
$$

where $N$ is the gap state density, $\phi$ the electrostatic potential in the depletion layer. Another point should be noted here that the charge stored in region 3 , $Q_{3}$ should be the same at all voltage biases, since $\mathrm{d} \phi / \mathrm{d} x$ is vanished at $x=W$. This means that $\left.(\mathrm{d} \phi / \mathrm{d} x)\right|_{b}\left(\equiv Q_{3} / \epsilon\right)$ in eqn (11) is also a constant.

In order to derive the $C-V$ relation, we need to know the change of total charge under a small a.c. signal. Starting from Poissons equation, $\mathrm{d}^{2} \phi / \mathrm{d} x^{2}=-\rho(x) / \epsilon$, multiply both sides by $2(\mathrm{~d} \phi / \mathrm{d} x)$ and integrate from $x=a$ to 0 , the following equation is obtained:

$$
\left.\left(\frac{\mathrm{d} \phi}{\mathrm{d} x}\right)^{2}\right|_{0}-\left.\left(\frac{\mathrm{d} \phi}{\mathrm{d} x}\right)^{2}\right|_{a}=\frac{-2}{\epsilon} \int_{\phi(a)}^{\phi(a)-v_{p}} \rho(\phi) \mathrm{d} \phi
$$

The values of $\epsilon(\mathrm{d} \phi / \mathrm{d} x)$ at $x=0$ and $a$ correspond to the total charge $Q_{1}$ and $Q_{2}+Q_{3}$, respectively, where $Q_{\mathrm{t}}$ is the total space charge, $Q_{2}$ and $Q_{3}$ are the charges stored in region 2 and 3 , respectively. The integral in the right hand side has the order of $Q^{2}$ and we can define an equivalent charge, $Q_{109}$, such that:

$$
Q_{\text {leq }}^{2}=Q_{\mathrm{t}}^{2}-\left(Q_{2}+Q_{3}\right)^{2}=\frac{-2}{\epsilon} \int_{\phi(a)}^{\phi(a)-v_{p}} \rho(\phi) \mathrm{d} \phi .
$$

During the $C-V$ measurement, however, the situation is more complex as some deeper states can not respond to the a.c. signal. The possible charge distribution after bias increases to $V+d V$ is shown in Fig. 2(b) (---). From eqn (12), the variations of total charge can be separated into two parts, one due to $Q_{\text {ieq }}$ and the other due to $Q_{2}+Q_{3}$. As bias is increased, the second one will eventually dominate and $\Delta Q_{\mathrm{t}}$ can be approximated by:

$$
\Delta Q_{\mathrm{t}} \approx \Delta\left(Q_{2}+Q_{3}\right)
$$

The change in voltage can be obtained by rewritten eqn (11):

$$
V=\frac{q N}{2 \epsilon}\left[\left(b-a+\bar{x}_{3}\right)^{2}-\bar{x}_{3}^{2}\right]
$$

where $\bar{x}_{3}\left[\equiv Q_{3} /(q N)\right]$ is an equivalent width of region 3. The second term can be neglected at large reverse bias as compared to the first one. Since the emission rate for deep states depends exponentially on their activation energy, a new level $E_{\mathrm{j}}$ could be defined (Fig. 2a) such that all the states with energies above $E_{\mathrm{j}}$ and thus with very fast emission rates can respond to the a.c. measuring signal. $E_{\mathrm{j}}$ intersects $E_{\mathrm{f} n}$ at $x_{0}$ in region 3 . If the charge stored in the rear part of region $3\left(x_{0} \leqslant x \leqslant W\right)$ is defined as $Q_{4}$ and the charge density at $x_{0}$ is $N\left(x_{0}\right)$ then the capacitance $C$ measured using a.c. signal is given by:

$$
C=\frac{\epsilon}{x_{0}+\overline{x_{4}}},
$$

where $\overline{x_{4}}=Q_{4} /\left[q N\left(x_{0}\right)\right]$. Substituting back into eqn (11), we obtain:

$$
V \approx \frac{\epsilon q N}{2}\left[\left(\frac{1}{C}+\frac{1}{C_{0}}\right)^{2}\right]
$$

where $1 / C_{0} \equiv\left[\left(b-a+\overline{x_{3}}\right)-\left(x_{0}+\overline{x_{4}}\right)\right] / \epsilon$. For sufficiently large bias, $1 / C \gg 1 / C_{0}$, the $1 / C^{2}-V$ curve of an amorphous junction shows a linear dependence on bias voltage and is quite similar to a crystal junction.

Based on these results we can check the previous assumption that the electron current density $J_{n}$ changes slower than the factor $\exp \left(\Delta E_{\mathrm{c}} / k T\right)$. From the SRH model the recombination rate, $R(E)$, is expressed as:

$$
R(E)=N_{\mathrm{i}}(E) \mu_{p} \sigma_{p} \frac{\left(p n-n_{\mathrm{i}}^{2}\right)}{n+\bar{n}+b(p+\bar{p})} .
$$

The denominator is smallest within $a \leqslant x \leqslant b$ and independent of $x$. Therefore the generation rate, $G(x)$, in eqn (5) can be regarded as constant within $a \leqslant x \leqslant b$. This means that $J_{n}$ depends, at most, linearly on $x$ and should rise much slower than $\exp \left(\Delta E_{\mathrm{c}} / k T\right)$ which depends exponentially on $x^{2}$

At this stage, the influence of measuring frequency on the capacitance is clear. The variation of the measuring frequency will change the parameter $x_{0}$ in eqn (13) and thus affect the measured capacitance. However, at large reverse bias, $1 / C_{0}$ becomes so small the linear $1 / C^{2}-V$ curve is preserved. As defined before, the density $N$ obtained is independent of the a.c. frequency.

Finally, we should discuss the effects of dangling bonds which have two charged states and are not accounted for by the SRH model. Although the presence of two charged states render the SRH formulation inapplicable, the basic physical concept should exist. No matter what the nature of the gap state is, if the free carrier density is small enough to make the emission rate dominant, the electron occupation probability should be determined by the relative magnitudes of the electron and hole emission rate. This will make $f(E)$ independent of $x$ and ensure the existence of a constant charge region.

\section{EXPERIMENTS}

The a-Si: $\mathrm{H}^{+}-n-n^{+}$junctions were fabricated in a conventional capacitively coupled glow discharge system. Figure 3 shows the device structure. The substrate was precoated with $1800 \AA$ indium tin oxide (ITO) and $200 \AA$ tin oxide. The gas phase doping concentration was $0.3 \%$ for both $p^{+}$and $n^{+}$layer, with $1 \mathrm{ppm} \mathrm{PH}_{3}$ doping for the $n$ layer. During the deposition, the chamber pressure, substrate temperature, and if power were kept at 0.5 Torr, $250^{\circ} \mathrm{C}$, and $1 \mathrm{~W}$, respectively. The thickness of each layer was $500 \AA, 1.2 \mu \mathrm{m}$, and $500 \AA$. After the deposition, Al was evaporated through a metal mask. The final device has an area of $3 \times 10^{-2} \mathrm{~cm}^{2}$. 


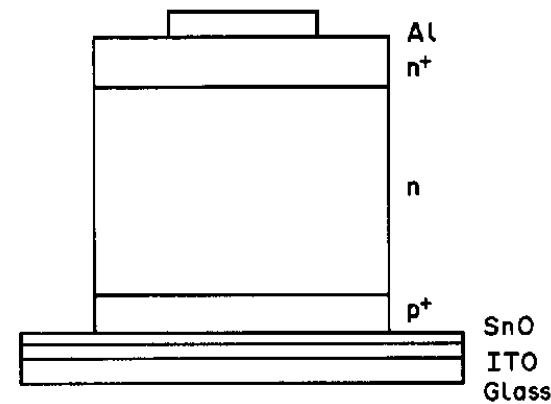

Fig. 3. The device structure of the $p^{+}-n$ junction used for the capacitance voltage measurement.

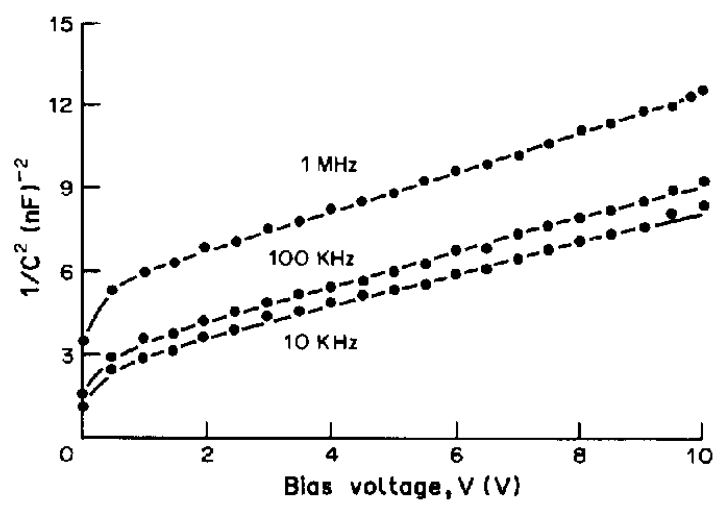

Fig. 4. The $1 / C^{2}$ vs bias voltage $V$ of an a $-\mathrm{Si}: \mathrm{H}^{+}-n$ junction with gas phase ratio of $1 \mathrm{ppm} \mathrm{PH}_{3} / \mathrm{SiH}_{4}$ in the $n$ layer.

Table 1. Summary of the net gap state density, $N$, for different $\mathrm{PH}_{3}$ doping in the $n$ layer

\begin{tabular}{ccc}
\hline $\mathrm{PH}_{3} / \mathrm{SiH}_{4}(\mathrm{ppm})$ & $\begin{array}{c}N \\
\left(\times 10^{16} \mathrm{~cm}^{-3}\right)\end{array}$ & $\begin{array}{c}\text { Density of state } \\
\left(\times 10^{17} \mathrm{~cm}^{-3} \mathrm{eV}^{-1}\right)\end{array}$ \\
\hline 1 & 1.8 & 0.6 \\
10 & 2.5 & 1.0 \\
100 & 4.9 & 2.5 \\
\hline
\end{tabular}

\section{RESULTS}

Figure 4 shows the $1 / C^{2}$ vs bias voltage curve of an a $-\mathrm{Si}: \mathrm{H}^{+}-n$ junction, with $1 \mathrm{ppm} \mathrm{PH}_{3}$ doped in the $n$ layer, measured at $10 \mathrm{~K}, 100 \mathrm{~K}$ and $1 \mathrm{MHz}$, respectively. The d.c. biases are changed slowly at approximately $0.25 \mathrm{~V} / \mathrm{min}$. As predicted from the theory, the slopes of the curves are independent of the a.c. measuring frequencies, since the measured charge density is determined by the d.c. bias voltage not those responded to the a.c. voltage. From the slope of the linear part of the curve, we obtain a net gap state density, $N$, of $1.8 \times 10^{16} \mathrm{~cm}^{-3}$ and the slopes were almost parallel for the three frequencies. Considering the difference between $E_{\mathrm{i}}^{\prime}$ and $E_{\mathrm{F}}$ being $\sim 0.3 \mathrm{eV}$ (estimating from the dark conductivity ratio), the density of state near $E_{\mathrm{i}}^{\prime}$ is approximately $6 \times 10^{16}$ $\mathrm{cm}^{-3} \mathrm{eV}^{-1}$ and is comparable to the results from DLTS. The capacitance decreases as the frequency is increased. This is because at higher frequency the $E_{\mathrm{f}}$ level became shallower and the distance $x_{0}$ will be increased to result in a lower capacitance. However, at low bias, the $1 / C^{2}-V$ curve deviates from the linear relation since the assumption of eqn (14) is violated. The net gap state density for the samples of different $\mathrm{PH}_{3}$ doping in the $n$ layers are summarized in Table 1. The densities were around $10^{16} \mathrm{~cm}^{-3}$ and increased as doping increased. Since the dark conductivity was aproximately $10^{-4}(\Omega-\mathrm{cm})^{-1}$ for doping higher than $40 \mathrm{ppm}$, the density of $100 \mathrm{ppm}$ doping is influenced by the free electron density.

\section{CONCLUSIONS}

Based on the SHR recombination model and carrier transport equation, we have reconsidered the results of $C-V$ measurements and find that the linear relation between $1 / C^{2}$ and $V$ is also preserved as in the crystalline case. Also the densities obtained from the slope are for those states situated between the equilibrium and the intrinsic Fermi-levels, not those responded to the a.c. signal.

\section{REFERENCES}

1. A. M. Goodman, J. appl. Phys. 34, 329 (1963).

2. J. D. Cohen, Semiconductors and Semimetals (Edited by J. l. Pankove), vol. 21, part C, chap. 2.

3. G. I. Roberts and C. R. Crowell, J. appl. Phys. 41, 1767 (1970).

4. P. Viktorovitch and D. Jousse, J. Non-cryst. Solids 35/36, 587 (1980)

5. D. V. Lang, J. D. Cohen and J. P. Harbison, Phys. Rev. 25B, 5285 (1982).

6. R. N. Hall, Phys. Rev. 87, 387 (1952).

7. W. Shockley and W. T. Read, Phys. Rev. 87, 835 (1952).

8. C. H. Henry, R. A. Rogan and F. R. Merritt, J. appl. Phys. 49, 3530 (1978).

9. J. D. Cohen and D. V. Lang, Phys. Rev. 25B, 5321 (1982).

10. S. M. Sze, Physics of Semiconductor Devices, 2nd edn (1981). 